\title{
TERMOGRAFIA APLICADA AO FENÔMENO DE RAYNAUD: ARTIGO DE REVISÃO BIBLIOMÉTRICA
}

\author{
Catia Terezinha Heimbecher ${ }^{1,2}$, Leandra Ulbricht ${ }^{1}$
}

1 Programa de Pós-Graduação em Engenharia Biomédica/Universidade Tecnológica do Paraná (UTFPR), Curitiba, Brasil. ${ }^{2}$ Faculdades Integradas Santa Cruz de Curitiba - Faresc, Curitiba, Brasil.

Resumo: A termografia é um método não invasivo, sem contato físico, não apresentando riscos para o paciente ou para o aplicador. Os sensores detectam a quantidade de radiação infravermelha apresentada na superfície do corpo. Os objetivos deste estudo foram identificar protocolos para: a) verificar os que tiveram resultados positivos na detecção do Fenômeno de Raynaud e b) identificar os pontos em comum entre eles. Este é um estudo exploratório que utilizou como ferramenta a bibliometria, sendo incluídos os artigos publicados entre 2013 a 2016. Foi realizada uma análise descritiva onde os artigos foram categorizados e descritos conforme os dados de publicação, autoria e equipamento utilizado. Os resultados apontam uma variedade de protocolos de utilização da termografia no estudo do Fenômeno de Raynaud e de seu comportamento na forma primária e secundária. Como conclusão identifica-se um grande uso da termografia na detecção de doenças do tecido conectivo. A maior parte destes estudos utiliza os seguintes critérios: Deve-se manter a sala entre $22^{\circ} \mathrm{C}$ e $23^{\circ} \mathrm{C}$ (graus Celsius). $\mathrm{O}$ estresse ao frio deve ser realizado com água entre $10^{\circ} \mathrm{C}$ a $20^{\circ} \mathrm{C}$ (graus Celsius). Acredita-se que um protocolo que adote os critérios listados baseados nas evidências dos estudos realizados, seja capaz de detectar o F.R.

Palavras-chave: Termografia; Fenômeno de Raynaud; Síndrome de Raynaud; Doença de Raynaud

\begin{abstract}
Thermography is a non-invasive method, without physical contact, presenting no risks to the patient or the applicator. The sensors detect the amount of infrared radiation displayed on the surface of the body. The objectives of this study were to identify protocols to: a) verify those that had positive results in the detection of the Raynaud Phenomenon and b) to identify the points in common between them. This is an exploratory study that used bibliometrics as a tool, and the articles published between 2013 to 2016 were included. A descriptive analysis was carried out where the articles were categorized and described according to the data of publication, authorship and equipment used. The results point to a variety of protocols for the use of thermography in the study of the Raynaud Phenomenon and its behavior in the primary and secondary forms. In conclusion, a great use of thermography in the detection of connective tissue diseases is identified. Most of these studies use the following criteria: The room should be maintained between $22^{\circ} \mathrm{C}$ and $23^{\circ} \mathrm{C}$ (degrees Celsius). Cold stress should be performed with water between $10^{\circ} \mathrm{C}$ and $20^{\circ} \mathrm{C}$ (degrees Celsius). It is believed that a protocol that adopts the listed criteria based on the evidences of the realized studies, is able to detect the F.R.
\end{abstract}

Keywords: Thermography; Raynaud's Phenomenon; Raynaud's Syndrome; Raynaud's disease 


\section{†PAJMT INTRODUÇÃO}

A termografia é um método não invasivo, sem necessidade de contato físico, não apresentando riscos para o paciente ou para o aplicador. Suas imagens apresentam-se de forma bidimensional e os sensores detectam a quantidade de radiação infravermelha apresentada na superfície do corpo., ${ }^{1,2}$

As câmeras termográficas utilizam sensores na faixa de comprimento de onda entre 0,75 a $14 \mu \mathrm{m}$ e convertem a radiação térmica em sinais elétricos. Inicialmente, as pesquisas eram na área militar, mas progressivamente mostraram que as temperaturas em algumas partes do corpo apresentam diferenças. Essa descoberta levou a tecnologia à área biomédica. ${ }^{3,4}$

Atualmente a termografia é usada para avaliar modalidades terapêuticas em conjunto com as informações clínicas ou associada a outros exames complementares, para auxiliar no estabelecimento de um diagnóstico clínico. ${ }^{2,3,5}$

No exame termográfico os locais de maior circulação sanguínea aparecem como pontos quentes, comumente relacionados à inflamação aguda. Por outro lado, pontos frios, representam baixa vascularização, indicando, por exemplo, necroses, tromboses ou inflamações crônicas. ${ }^{2,4}$

Uma das possíveis aplicações da tecnologia se refere ao estudo do Fenômeno de Raynaud (F.R.), onde a termografia avalia as diferenças de temperatura na superfície da pele podendo determinar o fluxo sanguíneo na microcirculação. ${ }^{5-14}$

Este fenômeno é resultante de uma vasoconstrição exagerada das artérias e arteríolas digitais que induzem alterações de cores na pele, podendo torná-la avermelhada, esbranquiçada ou azulada. Isto ocorre da região distal para proximal, ou seja, cianose distal, vasoconstrição central e vasodilatação proximal, atuando de maneira paroxística nos dedos das mãos, pés, nariz, lóbulos das orelhas e até mesmo lábios. ${ }^{15-18}$

O FR primário é também conhecido como idiopático, estando relacionado com história familiar por não se identificar uma doença de base. Tem como principal característica alterações funcionais sem danos nas paredes dos vasos. Em geral, as manifestações são brandas e simétricas. 12,16,17,19

A forma secundária apresenta início tardio, ou seja, em idade adulta, tendo como característica associação com doença de base, normalmente autoimune, e alterações estruturais nos vasos sanguíneos. ${ }^{15,19}$

Existem diversos protocolos para aquisição de imagens infravermelhas, incluindo o F.R. Contudo, as inúmeras diferenças metodológicas entre os estudos, causa insegurança na adoção de qualquer um deles. ${ }^{20}$

Assim, o objetivo deste estudo foi identificar os diversos protocolos para: a) verificar os que tiveram resultados positivos na detecção do F.R. e b) identificar os pontos em comum entre eles.

\section{METODOLOGIA}

Esta pesquisa trata de um estudo exploratório ${ }^{21}$ que utilizou como ferramenta a bibliometria. Este é o processo de evidenciação dos dados estatísticos de um conjunto definido de artigos gerando informação e conhecimento científico sobre um assunto específico que acontece pela contagem de documentos, visando identificar lacunas nas pesquisas de um determinado tema22.

Para este estudo foram incluídos os artigos publicados entre 2013 a 2016, com as palavras chave formando os algoritmos de busca: Termografia AND Fenômeno de Raynaud, Termografia AND Síndrome de Raynaud, Termografia AND Doença de Raynaud, phenom* Raynaud AND thermography, Raynaud's syndrome AND thermography, Raynaud's disease AND thermography - Fenómeno de Raynaud AND termografia, síndrome de Raynaud AND termografía, enfermedad de Raynaud AND termografía fenomeno di Raynaud AND termografia, síndrome di Raynaud, malattia di Raynaud AND termografia.

A busca foi executada nas bases Web of Science, PubMed, IEEE, Scopus e Elsevier, todas alojadas no Portal de Periódicos da CAPES. Os artigos então passaram pelo processo de filtragem, conforme Figura 1, de títulos e resumos onde foram descartados os artigos com títulos repetidos e selecionados os artigos aderentes ao tema e que apresentassem protocolo de avaliação. Após as exclusões foi gerado o portfólio bibliográfico.

O portfólio final foi composto de 15 artigos nos quais foram realizadas as análises métricas com o auxílio das ferramentas Mendeley (Versão 1.17.9 Mendeley Ltda.) e Microsoft Excel (2007).

A partir destas informações, foi realizada uma análise descritiva onde os artigos foram categorizados e descritos conforme país de publicação, autor que mais participa de pesquisas com este tema, identificação da revista com maior número de publicações, câmeras termográficas mais utilizadas nos estudos. Posteriormente foi realizada a análise de conteúdo, focando na identificação de diversas características dos protocolos encontrados para responder aos objetivos desta pesquisa. 


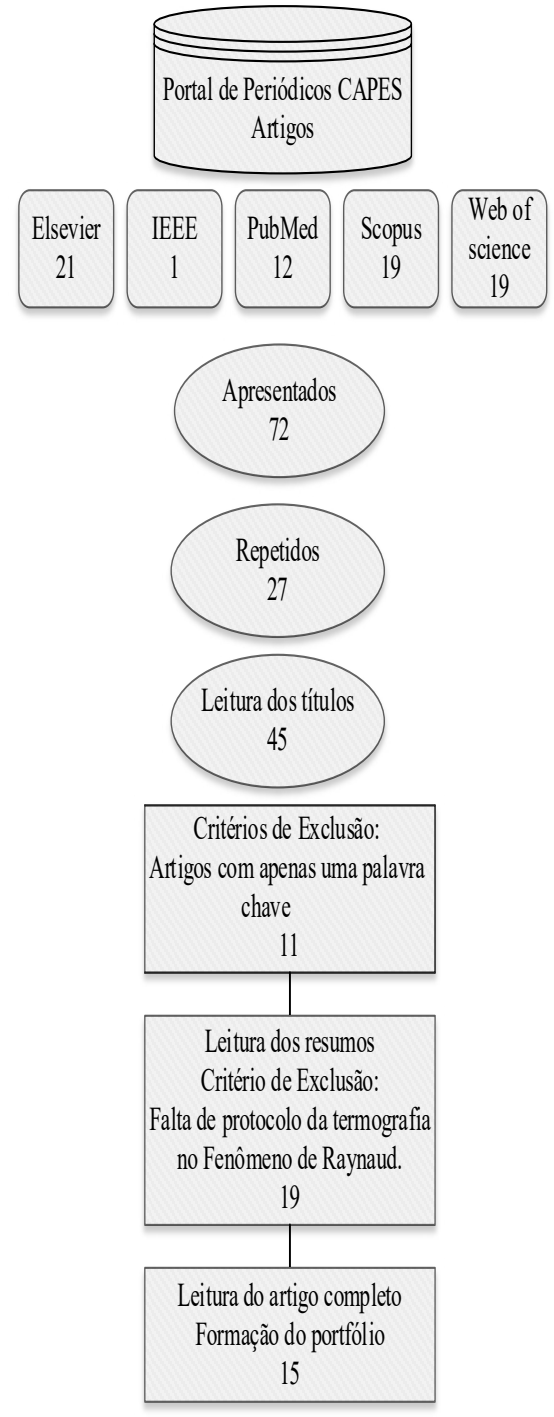

Figura 1 Procedimento metodológico

\section{RESULTADOS}

Houve retorno de artigos com o algoritmo redigido em inglês e apenas um com o algoritmo redigido em espanhol.

A Inglaterra produziu 33,33\% com 5 publicações, sendo o principal país a desenvolver pesquisa utilizando a termografia no estudo do Fenômeno de Raynaud no período entre 2013 a 2016. Em seguida, estão a Coréia, Japão e Itália 13,32\% cada país sendo 2 estudos cada e por fim Polônia, Estados Unidos da América (EUA), Rússia e Argentina com 6,66\% cada país o que representa 1 estudo cada país.

Dos países em questão, o pesquisador que mais publica é John Pauling, que está presente em 28,56\% das publicações, seguido por McHuggh e Shipley com $21,14 \%$ dos artigos. Vasta e Scolnik com 14,28\% e os demais autores contribuíram com uma publicação compondo 7,14\%. Estes autores colaboram com os artigos como autores principais ou coautores.

A principal revista a publicar os artigos selecionados é a Rheumatic International que é um periódico independente que reflete o progresso mundial na pesquisa, diagnóstico e tratamento nas várias doenças reumáticas. Seu fator de impacto é 1.702 e a editora é Springer Berlim Heidelberg.

A população estudada foi categorizada em grupos com FR primário e secundário, grupos controle, acrocianose, fibromialgia e esclerose sistêmica e voluntários classificados como grupo com "dedos gelados" que não foram classificados como reaquecimento normal, porém, sem confirmação de FR mas que apresentaram gradiente térmico $\geq-1^{\circ} \mathrm{C}$

A média amostral da população que participou dos estudos está descrita na Tabela 1.

TABELA 1 Apresentação de mínimo, média e máxima dos voluntários apresentados nos artigos selecionados.

\begin{tabular}{|r|r|r|r|r|}
\hline & N & Mínimo & Máximo & Média \\
\hline FR1 $^{\circ}$ & 5 & 20 & 85 & 43,60 \\
\hline FR2 $^{\circ}$ & 8 & 10 & 33 & 21,50 \\
\hline Controle & 8 & 13 & 146 & 68,13 \\
\hline Acrocianose & 1 & 8 & 8 & 8,00 \\
\hline Fibromialgia & 1 & 43 & 43 & 43,00 \\
\hline $\begin{array}{r}\text { Esclerose } \\
\text { Sistêmica }\end{array}$ & 2 & 22 & 28 & 25,00 \\
\hline $\begin{array}{r}\text { Dedos } \\
\text { gelados }\end{array}$ & 1 & 29 & 29 & 29,00 \\
\hline
\end{tabular}




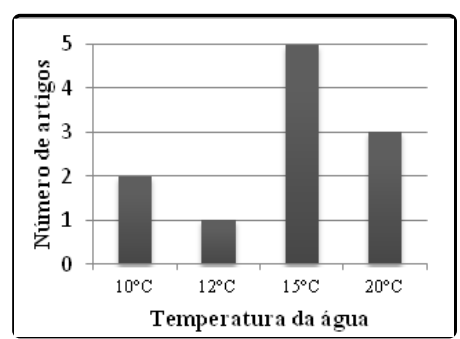

(A)

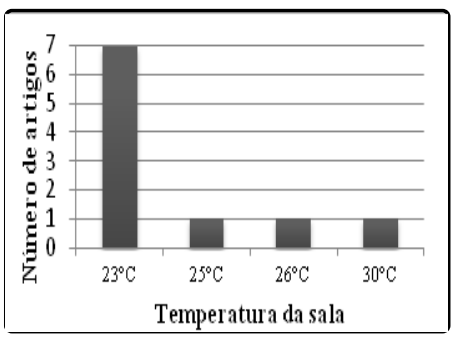

(B)

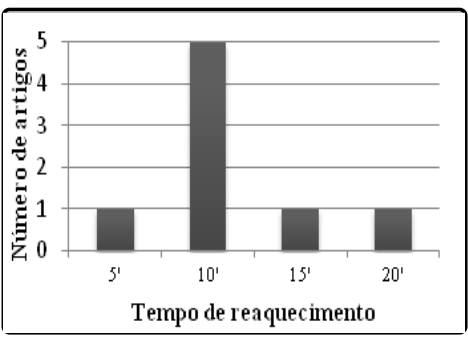

(C)

Figura 2. (A)Temperaturaura da água utilizada nas pesquisas. (B) Temperatura da sala em que os testes de provocação ao frio foram realizadas. (C) Tempo de reaquecimento das mãos após provocação ao frio em grupos controle quando não há presença do Fenômeno de Raynaud. Valores apresentados em graus Celsius $\left({ }^{\circ} \mathrm{C}\right)$ para temperatura e minutos para tempo.

Destaca-se que praticamente $35,70 \%$ dos artigos não identificou o equipamento utilizado.

Entre os artigos que traziam esta informação, o sistema FLIR com software CTHERM-versão 2.4 para análise das imagens, foi o mais utilizado, totalizando 28,6\% dos artigos. As demais câmeras aparecem em apenas um artigo cada somando 35,7\%, sendo elas: IRIS- XP® Seul, TH3 106ME, NEC San-ei instruments, Tóquio-Japão, Sistema VIGO - V 20II, Thermo-TRACER 3107 ME, NEC, Tóquio-Japão, TThermoteknik TTK versão 2.5, Thermoteknik TTX, AGEMA thermovision 870, AGEMA thermovision 570, FLIR thermacam sc500.

A distância entre a câmera e a região a ser termicamente registrada é descrita apenas em 2 estudos e são de $1,5 \mathrm{~m}^{10} \mathrm{e}$

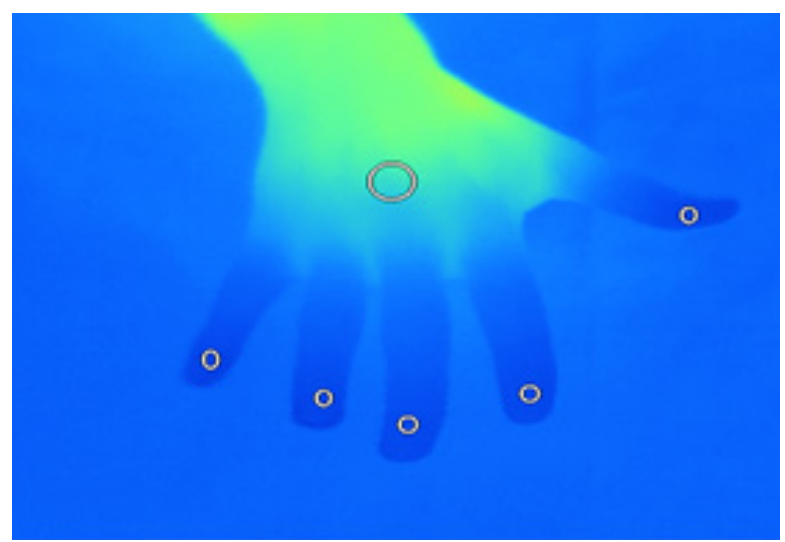

Figura 3 DDD - Diferença Distal Dorsal calculada pelo gradiente de temperatura entre as falanges distais dos metacarpos e dorso da mão.
$0,80 \mathrm{~m}^{12}$

Os Protocolos para aquisição de imagens infravermelhas envolvem: a preparação da área para a tomada de imagens, a adequação do local, distância entre a região e a câmera, escala térmica e posição do sujeito na aquisição de imagem. ${ }^{20}$

O cold stress (estresse ao frio) está presente na metodologia da aplicação da termografia no estudo do F.R. na maior parte dos artigos (80,92\%).

A Figura 2, apresenta aspectos relacionados a temperatura da água para o cold stress, temperatura do ambiente para a coleta dos dados e tempo de recuperação após o cold stress.

A maior parte dos artigos não utilizava a temperatura aferida, $76,92 \%$ utilizam a Diferença Distal Dorsal (DDD) para avaliar os resultados referentes as diferenças de temperatura e reaquecimento das mãos. A DDD é calculada pelo gradiente de temperatura entre as extremidades dos dedos e a região dorsal da mão (Figura 3). Nos demais, a análise foi realizada por regressão logistica ou não há indicação.

Quanto aos objetivos dos estudos, 19,04\% se propôs a comparar doenças do tecido conectivo (Figura 4). 


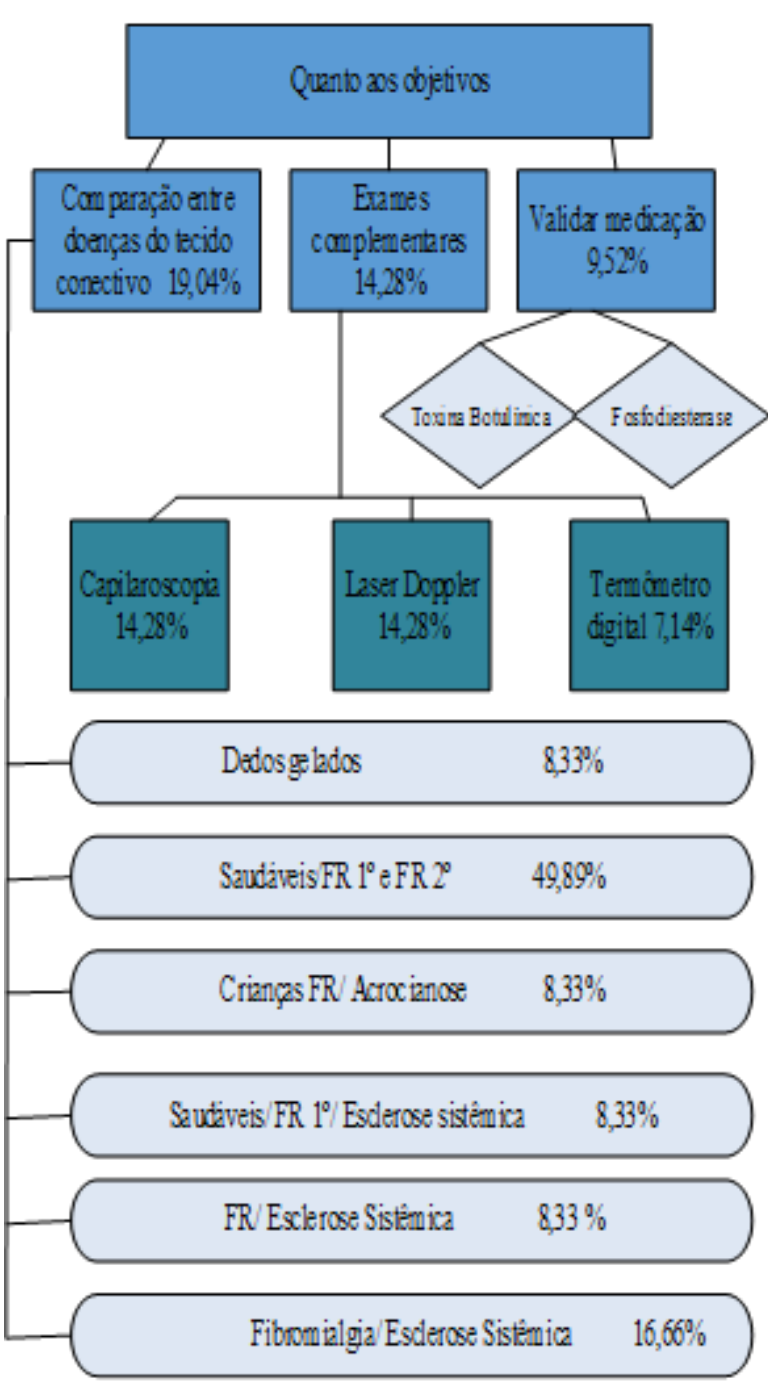

Figura 4. Objetivos dos artigos categorizados em quatro grupos, subdivididos nas áreas de desenvolvimento das pesquisas.

\section{DISCUSSÃo}

Os resultados desta revisão apontam a variedade de protocolos para estudo do Fenômeno de Raynaud e de seu comportamento na forma primária e nas situações de esclerodermia, acrocianose e fibromialgia.

Apenas dois estudos mostram a distância entre as mãos e a câmera ${ }^{10,12}$, porém este é um aspecto relevante no processo de reprodutibilidade e deve ser considerado ao se estabelecer um protocolo de aplicação da termografia no FR.

Entre os estudos com o cold stress, foram utilizadas diferentes temperaturas da água para se obter a vasoconstrição $\left(10^{\circ} \mathrm{C}, 12^{\circ} \mathrm{C}, 15^{\circ} \mathrm{C}\right.$ ou $\left.20^{\circ} \mathrm{C}\right)$. Esta temperatura vai estar relacionada ao tempo de resposta que variou entre cinco minutos, com

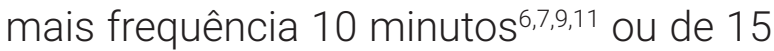
minutos ${ }^{13}$ em grupo controle, ou seja, voluntários que não apresentam o diagnóstico clínico de FR

A termografia se mostrou eficiente para comparar os resultados do uso de medicações, indicando que houve redução entre as crises de vasoconstrição prolongada antes e depois do tratamento. ${ }^{8,23}$

Uma das formas mais comuns de estudar o comportamento térmico no FR foi através da DDD, sendo delimitadas as regiões de falanges ditais e região de média de metacarpo para cálculo do gradiente térmico. ${ }^{5-7,9,11-14}$

Há descrição de dimensões de análise sendo de $1 \mathrm{~mm}$ para falanges e $2-3 \mathrm{~mm}$ em região palmar e dorsal. ${ }^{28}$

Para o estudo de Horikoshi6 no grupo controle, a DDD média foi positiva, ou seja, a temperatura da prega ungueal foi maior que a região do metacarpo exceto para 0 e 3 min após o estresse ao frio. Em contrapartida, os voluntários diagnosticados com FR, estiveram com DDD sempre negativa sendo que muitos não recuperaram a temperatura mesmo após 30 minutos de testes.

Para Chlebicka e Scolnik ${ }^{5,7}$, deve-se suspeitar de FR quando a diferença entre a extremidade dos dedos e o metacarpo for $\geq-3^{\circ} \mathrm{C}$, sugerindo que se faça um valor de corte para diferenças $\geq-1^{\circ} \mathrm{C}$ considerados como dedos "gelados" e não normais. No fenômeno de Raynaud, o DDD é tipicamente negativo com valores de $-1^{\circ} \mathrm{C}$ a $-3^{\circ} \mathrm{C}$ ou mais.

Scolnik também descreve que uma história de aumento da sensibilidade ao frio com a presença de mudanças de cor dos dedos é considerada suficiente para fazer 
(раjмт um diagnóstico clinico "definitivo" de FR. Porém, em seus estudos, também exigiu a ausência de características clínicas de uma doença reumática subjacente. ${ }^{7}$

Ismail ${ }^{10}$ verificou que o método de estimação direta dos parâmetros de resposta dinâmica em circuito fechado, baseado na análise do domínio do tempo, fornece uma descrição efetiva das diferenças funcionais entre pacientes e controles saudáveis, na termorregulação de dedos.

Para ele essas diferenças são suficientemente sólidas para apoiar um diagnóstico diferencial adequado da doença com base em dados de imagens térmicas e a avaliação da deficiência funcional após a estimulação térmica. Esta pesquisa corrobora com os estudos de Kolesov, ao afirmar que a temperatura mínima dos dedos de ambas as mãos é restabelecida aos 10 minutos após o resfriamento padrão. ${ }^{10,11}$

Lim e Allen ${ }^{13,29}$ acrescentam em suas análises, que se deve excluir o polegar, por estes serem poupados no FR primário e secundário, assim como o $5^{\circ}$ dedo, por ser pouco discriminatório em função do seu tamanho.

Pauling ${ }^{30}$ relata que polegares não favorecem a diferença entre FR e esclerodermia sistêmica. Além disso, propõe que seja verificada a temperatura central com termômetro infravermelho antes e depois de aclimatação de 30 minutos, sugerindo que esta aclimatação pode ser modificada em função da região e clima.

Schlanger utiliza também aclimatação com temperatura constante de $23^{\circ} \mathrm{C}$ com mãos livres de contato ou outros objetos. ${ }^{31}$

Para Pauling, o voluntário deve chegar antecipadamente e com tempo para exame evitando estar estressado. Deve evitar o consumo de cafeína, chocolate e similares 2 horas antes do teste, sendo permitida apenas água e/ou suco. Propõe que o voluntário não realize atividade física 2 horas antes do estudo e que não use hidratante, talco ou esmalte para unhas. Neste estudo, as imagens foram registradas a cada 5 minutos durante 30 minutos. Padronização utilizada também por Alen e Lim. 13,30,28

Pauling refere que estresse ao frio utilizando a água em temperatura de $20^{\circ} \mathrm{C}$, foi alta para o estudo proposto e sugere que novos testes sejam feitos a temperaturas menores. Sugere também o tempo de latência para recuperação no reaquecimento, conforme trabalho desenvolvido por Allen em 2014. O primeiro registro das imagens é feito no início do estudo e 10 minutos após estresse ao frio usando também o DDD para comparar os valores de cada dedo com a palma da mão..$^{30,13}$

Quanto à comparação entre a termografia e outros métodos não invasivos e sem contato físico estão a capilaroscopia que se baseia no reconhecimento de padrões para a avaliação morfológica, dimensão e densidade, levando em consideração, o número de capilares por área $(\mathrm{mm})$. Foi considerado normal 10 capilares por $\mathrm{mm}^{24,25}$

No FR primário, as manifestações são brandas e simétricas podendo resultar em capilaroscopia normal. ${ }^{19}$

Outro método é o laser doppler que utiliza fonte de luz laser monocromática para medir a velocidade de não homogeneidades no fluxo. ${ }^{26}$

Na utilização destes exames complementares na comparação com a termografia, esta complementa e expande as informações obtidas através do exame clínico e de outros métodos instrumentais de investigação especialmente nos estágios iniciais do desenvolvimento das doenças, quando os métodos de diagnóstico estruturais ainda não registraram mudanças significativas da corrente microvascular. Assim, indica que a termografia deve ser utilizada no diagnóstico e tratamento 
deste fenômeno. ${ }^{11}$

Na comparacação comportamento em relação às doenças do tecido conectivo, Ismail reforça a importância do diagnóstico diferencial citando a termografia neste processo. ${ }^{10}$

No caso da esclerose sistêmica, também conhecida por esclerodermia, há fibrose, ou seja, aumento excessivo do tecido conjuntivo de reparação disseminado no corpo todo. Ela se caracteriza por inflamação crônica compatível com doença autoimune, lesão de vasos pequenos e fibrose de região intersticial e perivascular com aspecto progressivo em indivíduos geneticamente predispostos. A poliartralgia e o Fenômeno de Raynaud são os primeiros sintomas, sendo que na maioria das vezes as dores articulares surgem depois do Fenômeno de Raynaud ${ }^{15,27}$ o que demonstra a importância de sua identificação precoce.

\section{CONCLUSÃO}

Os protocolos apresentados nesta revisão apontam para o uso da termografia no acompanhamento e controle de parâmetros térmicos na aplicação de novas medicações para controle do FR sendo um instrumento seguro e confiável nesta aplicação.

São apresentados também estudos que mostram que a termografia sendo utilizada em comparação a outros exames complementares, pode apresentar alterações térmicas, ainda não detectadas pela capilaroscopia.

Um grande uso da termografia tem sido na detecção de doenças do tecido conectivo comparando grupos diagnosticados seja com Fenômeno de Raynaud primário ou secundário presente em voluntários portadores de esclerose sistêmica, fibro- mialgia e grupo controle.

O método mais utilizado na interpretação destes resultados foi a utilização da DDD. Porém, alguns estudos não foram conclusivos na diferenciação entre FR primário e secundário. Contudo, existe diferença no reaquecimento pós-estresse ao frio, onde o grupo controle apresenta maior temperatura e o reaquecimento acontece em tempo menor.

Neste ponto, a maior parte dos autores apresenta um tempo de reaquecimento entre 10 minutos no grupo controle.

A maior parte destes estudos utiliza os seguintes critérios: Deve-se manter a sala entre $22^{\circ} \mathrm{C}$ e $23^{\circ} \mathrm{C}$ (graus Celsius). O estresse ao frio deve ser realizado com água entre $10^{\circ} \mathrm{C}$ a $20^{\circ} \mathrm{C}$ (graus Celsius).

Por fim, os estudos sugerem que a coleta pós-estresse ao frio deve ser realizada com tempo com variação de 10 a 30 minutos.

Acredita-se que um protocolo que adote os critérios listados baseados nas evidências dos estudos realizados, seja capaz de detectar o F.R.

\section{REFERÊNCIAS}

1. Ramirez-Arbeldez LM, Jiménez-dáiz KT, CorreaCastañeda AC, Giraldo-Restrepo JA, Fandiño HA. Protocolo de adquisición de imágenes diagnósticas por termografía infrarroja. Medicina \& Laboratorio. MedelinColombia; 2015 Apr;21(3-4):161-78.

2. Meira LF de, Krueger E, Neves EB, Nohama P, Souza MA. Termografia na área biomédica. Pan Am J Med Thermol. 2014;1(1):31-41.

3. Nola IA, Kolaric' D. Thermography in Biomedicine. In: 57 th International Symposium ELMAR-2015. Zagreb, Croatia; 2015. p. 28-30.

4. Sanches IJ, Gamba HR, Souza MA, NEVES EB, Nohama P. Fusão 3D de imagens de MRI/CT e termografia. Rev Bras Eng Biomed. 2013;29(3):298-308.

5. Chlebicka I, Matusiak JMAJ, Baran E, Szepietowski JC. Freezing fingers syndrome, primary and secondary 
- PAjMT Raynaud's Phenomenon: Characteristic features with hand thermography. Acta Derm Venereol. 2013;93(4):428-32.

6. Horikoshi M, Inokuma M, Matsubara E, Okada R, Takahashi R, Kobayashi S, et al. Uneven temperature among fingers after cold-water immersion of hands is a useful parameter to identify disturbed peripheral circulation. 2014; 77:423-4.

7. Scolnik M, Vasta B, Hart DJ, Shipley JA, McHugh NJ, PAULING JD. Symptoms of Raynaud's phenomenon (RP) in fibromyalgia syndrome are similar to those reported in primary RP despite differences in objective assessment of digital microvascular function and morphology. Rheumatology International [Internet]. Springer Berlin Heidelberg; 2016; Available from: http://link.springer. com/10.1007/s00296-016-3483-6

8. Motegi S, Yamada K, Tokio S, Uchiyama A, Kubota $Y$, Nakamura T, et al. Beneficial effect of botulinum toxin A on Raynaud's phenomenon in Japanese patients with systemic sclerosis: A prospective, case series study. J Dermatol. 2016;43(June 2015):56-62.

9. Cappella M, Culpo R, Sprocati M, Meneghel A, Zulian F, Martini G. Infrared thermography after cold challenge validation in diagnosis of Raynaud's Phenomenon in pediatric age. BMJ Journals Ann Reum Dis. 2015;

10. Ismail E, Orlando G, Corradini ML, Amerio P, Romani GL, Merla A. Differential diagnosis of Raynaud's phenomenon based on modeling of finger thermoregulation. Physiological measurement [Internet]. 2014;35(4):703-16. Available from: http://dx.doi. org/10.1088/0967-3334/35/4/703\%5Cnhttp://www.ncbi. nlm.nih.gov/pubmed/24622281

11. Kolesov SN. Thermalvision diagnosis of Raynaud's syndrome and its stages. J Opt Tecnol. 2015;478-86.

12. Scolnik M, Vasta B, HART D, Shipley JA, Brown S, Korendowych E, et al. Fenómeno de Raynaud: características clínicas reportadas por los pacientes y evalaución objetiva por termografía. In: 460 Congresso Argentino de Reumatologia. 2013.

13. Allen J, Young AL, Griffiths B. Subject Preparation and Thermal Acclimatization Prior to Mild Cold Challenge Testing Using Dynamic Thermal Imaging. Pan Am J Termology [Internet]. 2014;1(1):51-4. Available from: doi: http://dx.doi.org/10.18073/2358-4696/pajmt.v1n1p51-54

14. Anderson ME, Moore TL, Lunt M, Herrick AL. The "distal - dorsal difference": a thermographic parameter by which to differentiate between primary and secondary Raynaud's phenomenon. Oxford Univ Press journals. 2007;(August 2006):533-8.

15. Robbins SL, Cotran RS. Patologia:Bases Patológicas das Doenças. 8a ed. Ed. Elsevier, editor. Rio de Janeiro: 2010; 2010.

16. Vilella CT, Aznar CPS, Julià LG. El fenómeno de Raynaud. Medicina Clinica. 2009;132(18):712-8.
17. Gamarra Al, Arroyave DJ, Quintana G, Herrera FR, Matucci-Cerinic M. Historia del compromiso cutáneo de la esclerosis sistémica. Revista Colombiana de Reumatología. Bogotá - Colombia; 2013;20(3):155-70.

18. Silva I, Loureiro T, Almeida I, Mansilha A, Almeida R, Vasconcelos C. Fenómeno de Raynaud. Angiol e Cir Vasc. 2011;7(1):13-20.

19. Kayser C, Corrêa MJU, Andrade LEC. Fenômeno de Raynaud. Rev Bras Reumatol. 2009;49(1):48-63.

20. Roldan KE, Piedrahita MAO, Dario Benitez H. Design and implementation of a protocol for acquisition and processing of infrared images obtained from hands. Symposium of Signals, Images and Artificial Vision 2013, STSIVA 2013. 2013;1-7.

21. Gil AC. Como elaborar projetos de pesquisa. 4th ed. Atlas, editor. São Paulo; 2002.

22. Lacerda RTO, Ensslin L, Ensslin SR. Uma análise bibliométrica da literatura sobre estratégia e avaliação de desempenho. Revista Gestão e Produção. 2012; v.19, n 1.

23. Kamata Y, Minota S. Effects of phosphodiesterase type 5 inhibitors on Raynaud's phenomenon. Rheumatology international [Internet]. 2014;34(11):16236. Available from: http://link-1springer-1com-1pubmed. han.medunigraz.at/article/10.1007/s00296-014-3025-z/ fulltext.html

24. Souza EJ do R, Kayser C. Capilaroscopia periungueal: relevância para a prática reumatológica. Rev Bras Reumatol [Internet]. Elsevier Editora Ltda.; 2015;55(3):264-71. Available from: http://linkinghub. elsevier.com/retrieve/pii/S0482500414002046

25. Cutolo M, Sulli A, Smith V. How to perform and interpret capillaroscopy. Best Practice and Research: Clinical Rheumatology [Internet]. Elsevier Ltd; 2013;27(2):237-48. Available from: http://dx.doi. org/10.1016/j.berh.2013.03.001

26. Albrecht $\mathrm{H}-\mathrm{E}$, Borys $\mathrm{M}$, Damaschke $\mathrm{N}$, Tropea C. Laser Doppler and phase Doppler measurement techniques. Vol. 43, Cryogenics. 2003. p. 738.

27. Horimoto AMC, Costa IP. Sobreposição de esclerose sistêmica e artrite reumatoide: uma entidade clínica distinta? Revista Brasileira de Reumatologia [Internet]. Elsevier Editora Ltda.; 2016;56(4):287-98. Available from: $\quad$ http://linkinghub.elsevier.com/retrieve/pii/ S0482500415000303

28. Lim MJ, Kwon SR, Jung K, Joo K, Park S, Park W. Digital Thermography of the Fingers and Toes in Raynaud's Phenomenon. J Korean Med Sci. 2014; 29:502-6.

29. Lim MJ, Kwon SR, Jung KH, JOO K, PARK SG, PARK W. Infrared thermography of big toe in Raynaud's Phenomenon. J Gen Intern Med [Internet]. 2013;25(S3):205-567. Available from: http://link.springer. com/10.1007/s11606-010-1338-5 
30. Pauling JD, Flower V, Shipley JA, Harris ND, McHugh $\mathrm{NJ}$. Influence of the cold challenge on the discriminatory capacity of the digital distal-dorsal difference in the thermographic assessment of Raynaud's phenomenon. Microvasc Res [Internet]. Elsevier Inc.; 2011;82(3):3648. Available from: http://dx.doi.org/10.1016/j. mvr.2011.03.007

31. Schlanger O, Gschwandtner ME, Herberg K, Frohner $\mathrm{T}$, Schillinger $\mathrm{M}$, Koppensteiner $\mathrm{R}$, et al. Correlation of infrared thermography and skin perfusion in Raynaud patients and in healthy controls. Microvasc Res [Internet]. Elsevier Inc.; 2010;80(1):54-7. Available from: http:// dx.doi.org/10.1016/j.mvr.2010.01.010. 
